\title{
Oral presentations
}

gowns and gloves and eye protection, respectively. Only $22 \%$ of participants were preparing cytotoxic drugs in a designated room while 68\% used a dedicated room for administration to the patient. A cytotoxic spill kit was available in nearly half of the practices where the respondents worked. There was no association found between age, gender, time since graduation, university of graduation and type of practice and the use of recommended equipment for preparation of cytotoxic agents.

Although $80 \%$ of participants reported seeking training and advice from various sources, less than half of them reported appropriate training in the safe handling of hazardous substances of other members of staff indirectly involved.

\section{CONCLUSION}

The study suggests that, despite training and concerns with health and safety issues associated with manipulation of hazardous drugs, compliance with recommended protocols was generally poor amongst veterinary healthcare workers in first opinion UK practices.

\section{Prognostic significance of immunophenotype in canine acute myeloid leukaemia}

\section{Jennifer Lowe, Maciej Guzera, Joy Archer, Tim Williams}

Cambridge University, Department of Veterinary Medicine, Cambridge, UK

Acute myeloid leukaemia (AML) is known to carry a poor prognosis in dogs, but little is known of any prognostic factors related to this disease. Immunophenotype is of prognostic value in chronic lymphocytic leukaemia, and anaemia is associated with a poorer prognosis in acute leukaemias. This study investigated the use of immunophenotype, classified using flow cytometry, and other clinical parameters as prognostic indicators in canine AML.

Dogs diagnosed with $\mathrm{AML}$ on flow cytometry at one institution between 1st January 2005 and 1st January 2013 were reviewed and a follow up history was obtained. Dogs were excluded if the diagnosis was uncertain; there was no follow up history; were CD34 negative (indicating chronic leukaemia), or were negative for all three myeloid markers (CD14, myeloperoxidase [MPO] or neutrophil specific antibody [anti-neut]). The relationship between survival time and the following variables were analysed using log rank analysis: breed, immunophenotype (divided into the following groups: [CD14+, MPO+, anti-neut+], [CD14+, MPO+],
[CD14+], [MPO+ and/or anti-neut+]), treatment type (palliative only, prednisolone or various chemotherapy protocols), haematological parameters at diagnosis and clinical signs. Survival time was calculated from the date of flow cytometric diagnosis to the date of natural death or euthanasia. Data are presented as median [range] and statistical significance was defined as $\mathrm{P}<0.05$.

Twenty four dogs met the inclusion criteria with an age of 7 [1-14] years; Labrador Retrievers, Golden Retrievers and German Shepherds were the most commonly represented breeds. Survival time was 3 [0-29] days. No association was identified between the immunophenotype of the disease and the survival time. However dogs with anaemia $(\mathrm{HCT}<36 \%)$ at the time of diagnosis had significantly shorter survival times compared with dogs with a HCT $\geq 36 \%$ (3[028] days, $n=21$ vs. 21[6-29] days, $n=3, P=0.025)$. Dogs presenting with gastrointestinal signs had significantly shorter survival times than those with no signs (3[0-6] days, $n=10$ vs 4[1-29] days, $n=11 ; P=0.03)$. Four cases presented with shifting lameness. Dogs surviving $\geq 3$ days were compared by treatment type; those treated with prednisolone had longer survival times compared to those treated with various chemotherapy protocols (21[3-28] days, $n=3$ vs. 7[3-29] days, $n=6 ; P=0.041)$.

Immunophenotype does not appear to be associated with prognosis in canine AML. However anaemia at the time of diagnosis and gastrointestinal signs are associated with a poorer prognosis in dogs with $\mathrm{AML}$, although prognosis is invariably poor.

\section{Evaluation of a lomustine based chemotherapy protocol (LOP) to treat canine T-cell lymphoma in 7 dogs}

\section{Charles Pittaway, Sarah Mason, Aleksandra Marcinowska, Antonio Giulano, Jane Dobson}

Department of Veterinary Medicine, Cambridge University, Cambridge, UK

High grade T-cell lymphoma in dogs is associated with a poorer prognosis compared to the B-cell phenotype when treated with $\mathrm{COP}$ and $\mathrm{CHOP}$ based protocols. One study previously reported that lomustine based protocols were more effective at treating multicentric and mediastinal canine T-cell lymphoma with a similar response rate and a longer progression free interval when compared with a CHOP protocol.

This retrospective study evaluated cases diagnosed with T-cell lymphoma at a single referral institution and that were subsequently treated with a LOP (lomustine, vincristine and prednisolone) protocol. Mean lomustine dose was $57 \mathrm{mg} / \mathrm{m}^{2}$ (range $40-65 \mathrm{mg} / \mathrm{m}^{2}$ ) every 4 weeks, and mean vincristine dose was $0.66 \mathrm{mg} / \mathrm{m}^{2}$ (range $0.5-0.7 \mathrm{mg} / \mathrm{m}^{2}$ ) weekly for 4 weeks then every 2 weeks. Response rate was recorded and progression free interval (PFI) was calculated from the date of first chemotherapy administration until recurrence of disease. Time taken before change in protocol due to adverse effects was calculated where appropriate and treatment delays and hospitalisation events due to adverse effects were recorded. Seven cases of high grade T cell lymphoma were included (3 multicentric, 2 mediastinal, 
1 abdominal and 1 nasopharyngeal). All 7 dogs were male, with a mean age of 6 years and were of assorted breeds with variation in body surface area (BSA) from 0.63 to $1.55 \mathrm{~m}^{2}$. The overall response rate was 100\% (100\% complete response). The median PFI was 93 days (range $43-730$ days) and median overall survival time was 146 days (range 51 to $730+$ days). Four dogs required changes in their protocol due to adverse effects (1 due to neutropenia associated with vincristine, 2 due to elevations in liver enzymes and 1 due to owner perceived reduction in dog's quality of life). The median time taken to protocol change was 76 days (range 27-139 days). One dog had to be hospitalised and 3 dogs required treatment delays due to neutropenia. $\mathrm{PFI}$ is similar to that previously reported with lomustine based chemotherapy protocols but these results show an improved complete response rate when compared to previous studies.

\section{Risk of development of keratoconjunctivitis sicca in dogs with sinonasal and maxillary tumours treated with radiotherapy}

\section{Irina Gramer, Laura Blackwood}

University of Liverpool, Small Animal Teaching Hospital, Neston, Wirral, UK

\section{INTRODUCTION}

Radiotherapy $(\mathrm{RT})$ is commonly used to treat canine sinonasal and maxillary tumours. However, these tumours are adjacent to important, normal organs at risk (OAR) including ocular structures, the lacrimal gland, and forebrain. KCS is a recognized complication of sinonasal/maxillary irradiation, but its frequency is unknown. This retrospective study aimed to determine the frequency of KCS, period of risk and possible risk factors.

\section{MATERIAL AND METHODS}

A total of 59 dogs treated with fractionated RT for sinonasal and maxillary tumours were retrospectively evaluated (2010 to 2014). In all patients RT was performed using a computer generated treatment plan at 6MV or 10MV. KCS was defined as reduced tear production ( $<15 \mathrm{~mm}$ ) on Schirmer tear test (STT). Reduced tear production was categorized into two groups: 1) mild-moderate KCS (5-15 mm) and 2) severe KCS
$(<5 \mathrm{~mm})$. The degree of KCS was correlated with the dose to the adjacent eye, as the lacrimal gland is not routinely contoured as an OAR. All dogs received artificial tears once an abnormal STT was documented.

\section{RESULTS}

Among the 59 dogs recruited, 23 different breeds were represented with a median age of nine years (3-14 years). There were fourteen different tumour types. Patients had a median follow-up time of 204 days (40-836 days). The dose to the adjacent eye ranged from 4-49Gy (mean 29.7Gy). KCS developed in $27.9 \%$ of patients with severe KCS in $15 \%$. Mild to moderate KCS was apparent in five (right eye) and eight (left) cases. Severe KCS developed in seven (right) and eight cases (left). In five dogs both eyes developed KCS. Dogs in which the ipsilateral eye received an RT dose over 35Gy were more likely to develop KCS $(p=0.002)$. The median duration from RT start to KCS detection was 82 days (22283 days). In this study population no other risk factors, besides RT dose, were identified.

\section{CONCLUSION}

Using the dose to the adjacent eye as an indicator, this study identified a cut-off RT dose of 35Gy after which dogs were significantly more likely to develop KCS. This occurred as early as 22 days and as late as 283 days, warranting regular monitoring to assure adequate treatment to prevent secondary corneal ulceration.

\section{Poor performance of routine} abdominal ultrasound in the detection of visceral metastasis in a uniform population of dogs with high-risk mast cell tumours

\section{Evi Pecceu, Juan Carlos Serra, Chiara Piccinelli, Ian Handel, Elspeth Milne, Jessica Lawrence}

Edinburgh University, Royal (Dick) School of Veterinary Studies, Edinburgh, UK

Conflicting evidence exists regarding the importance of routine abdominal ultrasound (AUS) with hepatic and splenic aspiration cytology during staging of canine mast cell tumours (MCTs) but it is considered standard and vital in dogs at high risk for metastasis. Our primary hypothesis was that despite selecting for dogs with high-risk MCT, AUS is poorly predictive of visceral metastasis. Our secondary hypothesis was that routine cytology in dogs with high-risk MCT reveals metastasis in fewer than 20\% of high-risk cases. The objective of this study was to correlate ultrasonographic changes and cytologic findings during initial staging in dogs with strictly defined high-risk MCTs.

Dogs were included if they were diagnosed with a high-risk MCT between March 2013 and March 2015 and if staging included regional lymph node (LN) assessment, AUS, and aspiration cytology of the liver and spleen. Inclusion criteria for dogs with high-risk MCTs were clearly defined. Standardised cytological criteria were used to define visceral MCT metastasis.

Of 42 dogs included, 2 (5\%) had liver abnormalities on AUS whereas 15 (36\%) had splenic abnormalities. In 4 dogs, 